\title{
Salt increases monocyte CCR2 expression and inflammatory responses in humans
}

\author{
Eliane F.E. Wenstedt, ${ }^{1}$ Sanne G.S. Verberk, ${ }^{2}$ Jeffrey Kroon, ${ }^{3}$ Annette E. Neele, ${ }^{4}$ \\ Jeroen Baardman, ${ }^{4}$ Nike Claessen, ${ }^{5}$ Özge T. Pasaoglu, ${ }^{1}$ Emma Rademaker, ${ }^{1}$ \\ Esmee M. Schrooten, ${ }^{1}$ Rosa D. Wouda, ${ }^{1}$ Menno P.J. de Winther, ${ }^{4,6}$ Jan Aten, ${ }^{5}$ Liffert Vogt, ${ }^{1}$ \\ Jan Van den Bossche $e^{2,4}$
}

${ }^{1}$ Amsterdam UMC, University of Amsterdam, Department of Internal Medicine, Section of Nephrology, Amsterdam Cardiovascular Sciences, Amsterdam, Netherlands. ${ }^{2}$ Amsterdam UMC, Vrije Universiteit Amsterdam, Department of Molecular Cell Biology and Immunology, Amsterdam Cardiovascular Sciences, Cancer Center Amsterdam, Amsterdam, Netherlands. ${ }^{3}$ Amsterdam UMC, University of Amsterdam, Department of Experimental Vascular Medicine, Amsterdam Cardiovascular Sciences, Amsterdam, Netherlands. ${ }^{4}$ Amsterdam UMC, University of Amsterdam, Department of Medical Biochemistry, Section of Experimental Vascular Biology, Amsterdam Cardiovascular Sciences, Amsterdam, Netherlands. ${ }^{5}$ Amsterdam UMC, University of Amsterdam, Department of Pathology, Amsterdam, Netherlands. ${ }^{6}$ Institute for Cardiovascular Prevention (IPEK), Munich, Germany.

Inflammation may play a role in the link between high salt intake and its deleterious consequences. However, it is unknown whether salt can induce proinflammatory priming of monocytes and macrophages in humans. We investigated the effects of salt on monocytes and macrophages in vitro and in vivo by performing a randomized crossover trial in which 11 healthy human subjects adhered to a 2-week low-salt and high-salt diet. We demonstrate that salt increases monocyte expression of CCR2, a chemokine receptor that mediates monocyte infiltration in inflammatory diseases. In line with this, we show a salt-induced increase of plasma MCP-1, transendothelial migration of monocytes, and skin macrophage density after high-salt diet. Macrophages demonstrate signs of an increased proinflammatory phenotype after salt exposure, as represented by boosted LPS-induced cytokine secretion of IL-6, TNF, and IL-10 in vitro, and by increased HLADR expression and decreased CD206 expression on skin macrophages after high-salt diet. Taken together, our data open up the possibility for inflammatory monocyte and macrophage responses as potential contributors to the deleterious effects of high salt intake.

Authorship note: SCSV and JK contributed equally to this work. LV and JVDB contributed equally to this work.

Conflict of interest: The authors have declared that no conflict of interest exists.

Copyright: (c) 2019, American Society for Clinical Investigation.

Submitted: June 5, 2019 Accepted: September 26, 2019 Published: November 1, 2019.

Reference information: /CI Insight. 2019;4(21):e130508. https://doi.org/10.1172/jci. insight.130508.

\section{Introduction}

Average salt $(\mathrm{NaCl})$ intake around the world substantially exceeds the amount ( $<5$ grams salt/day) that is recommended by WHO guidelines (1). High salt intake is associated with deleterious outcomes such as hypertension, kidney damage, and cardiovascular disease $(2,3)$. However, knowledge of potential explaining mechanisms remains incomplete. There might be a role for inflammation with regard to these mechanisms, since it has been suggested by several authors that inflammation plays a role in the previously mentioned diseases (4-9). Recent animal studies propose a role for specific phenotypes of circulating monocytes and macrophages, as well as their infiltration in various tissues such as the skin interstitium and the vessel wall $(6,10,11)$. In this regard, the potential inflammatory effect of salt on monocytes and macrophages is interesting to explore.

In humans, there is already some evidence that monocytes are affected by high salt (HS) $(12,13)$. Human monocytes are subdivided into 3 different subsets based on CD14/CD16 surface expression: classical $\mathrm{CD} 14^{++} / \mathrm{CD} 16^{-}$, nonclassical CD14 ${ }^{+} / \mathrm{CD} 16^{++}$, and intermediate CD $14^{++} / \mathrm{CD} 16^{+}$monocytes. Total peripheral monocyte counts and the percentage of intermediate monocytes increase upon high-salt diet (HSD) (12, 13). However, the effect of HS on the phenotype and function of those monocyte subsets remains unknown.

Infiltration of monocytes into tissues and transformation to macrophages may also be affected by salt. Whether salt increases monocyte infiltration and macrophage content in humans is unknown. With regard to the phenotype of macrophages, mouse studies reveal that salt promotes LPS-induced inflammatory macrophage responses, while inhibiting IL-4-induced antiinflammatory macrophage cues (14-16). Moreover, HS was shown to elicit a particular $\mathrm{M}(\mathrm{Na})$ gene expression profile in naive circulating human 


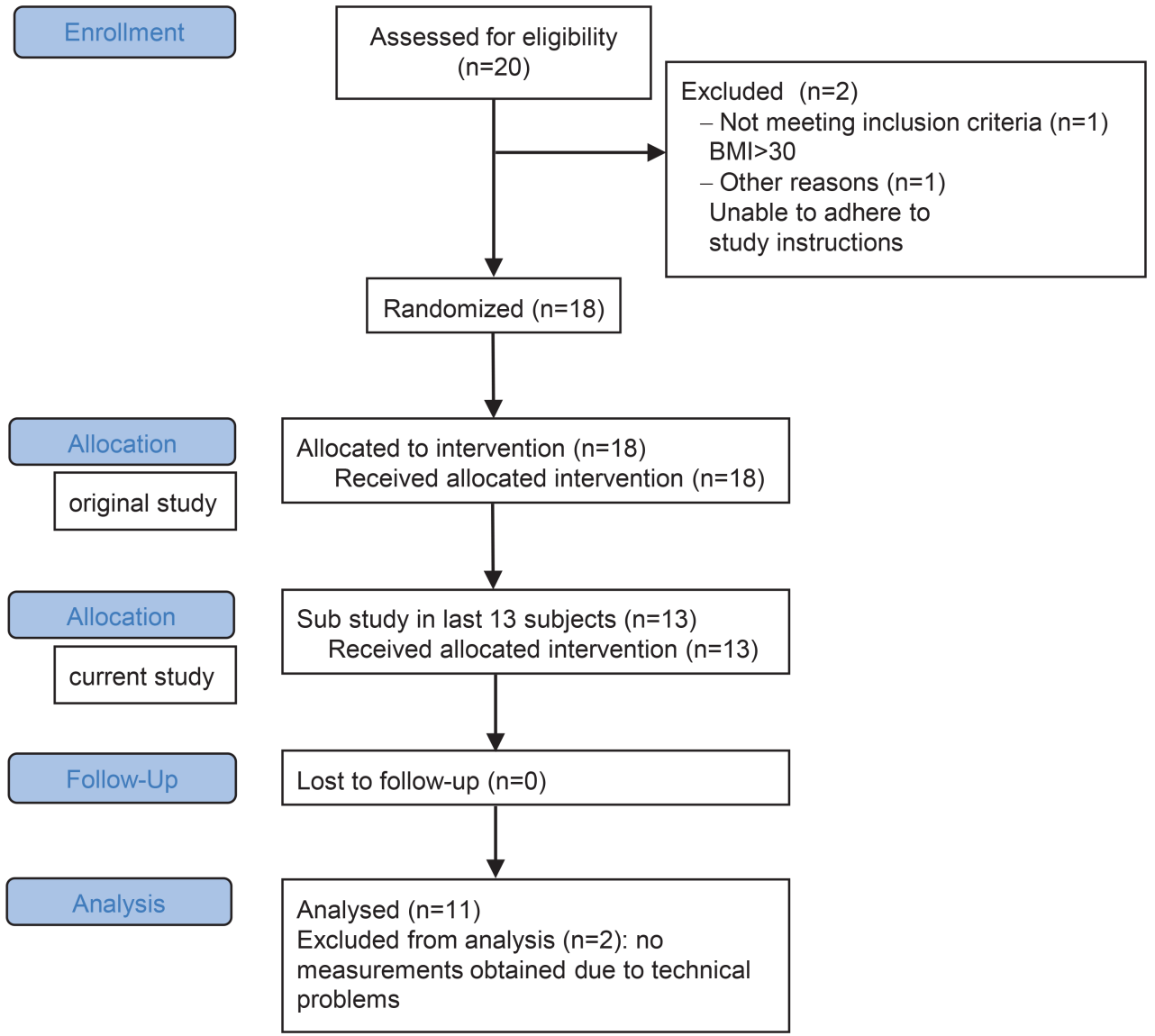

Figure 1. Flow chart for inclusion.

macrophages that were subjected to high-salt mediums in vitro (16). However, the effects of high salt intake on tissue macrophages have not been studied in human subjects. This is especially interesting, given the fact that high salt intake can increase tissue sodium concentrations to levels far exceeding those in the plasma, particularly in the skin (up to $180-190 \mathrm{mmol} / \mathrm{L})(17,18)$.

In this study, we set out to investigate the effect of HS on human monocytes and macrophages by salt exposure in vitro and by dietary salt loading. We hypothesized that HS primes monocytes and macrophages toward proinflammatory phenotypes and increases monocyte infiltration.

\section{Results}

HSD increases monocyte CCR2 expression. We studied the effect of salt intake on prevalence and phenotype of peripheral monocytes in 11 healthy subjects, who were included between September 2016 and April 2017 (Figure 1). Of the 11 included subjects, 5 subjects started with the HSD and 6 subjects started with the low-salt diet (LSD). The mean (SD) age was $28( \pm 5)$ years, and baseline blood pressure - determined at a screening visit before commencement of the diets — was $120( \pm 10) / 72( \pm 5) \mathrm{mmHg}$. Dietary salt consumption at time of screening (as estimated based on urinary sodium excretion) averaged 10 grams/day (176 mmol sodium). During HSD, average salt intake was 19 grams/day (324 mmol sodium) (Table 1). During LSD, average salt intake was 2 grams/day ( $32 \mathrm{mmol}$ sodium).

Systolic blood pressure, as well as body weight, increased after HSD (Table 1). HSD tended to increase the total number of circulating monocytes $(P=0.07)$ (Table 1$)$, with no difference in relative percentage of classical CD $14^{++} / \mathrm{CD} 16^{-}$, nonclassical CD $14^{+} / \mathrm{CD} 16^{++}$, and intermediate CD $14^{++} / \mathrm{CD} 16^{+}$monocyte subsets after LSD versus HSD (Figure 2, A and B). After HSD, CCR2 surface expression on classical monocytes strongly increased (Figure 2C). The effect of HS on monocyte phenotype was specific for CCR2, since other tested chemokine receptors (CCR5, CCR7, CX3CR1), scavenger receptors (CD36, SRA, CD163), and 
Table 1. Measured parameters after low-salt diet and after high-salt diet

\begin{tabular}{|c|c|c|c|}
\hline & Low-salt diet $(n=11)$ & High-salt diet $(n=11)$ & $P$ value \\
\hline Age (years) & $28(5)$ & $28(5)$ & - \\
\hline Weight (kg) & $78.5(10)$ & $80.5(10)$ & 0.0001 \\
\hline \multicolumn{4}{|l|}{ Office blood pressure } \\
\hline Systolic blood pressure $(\mathrm{mmHg})$ & $110(8)$ & $115(9)$ & 0.045 \\
\hline Diastolic blood pressure ( $\mathrm{mmHg}$ ) & $69(5)$ & $69(8)$ & 0.99 \\
\hline \multicolumn{4}{|l|}{ Plasma } \\
\hline $\mathrm{Hb}(\mathrm{mmol} / \mathrm{L})$ & $9.1(0.5)$ & $8.9(0.8)$ & 0.17 \\
\hline $\mathrm{Ht}(\mathrm{L} / \mathrm{L})$ & $0.4(0.02)$ & $0.4(0.03)$ & 0.88 \\
\hline MCV (fL) & $90(2.7)$ & $91(3.5)$ & 0.08 \\
\hline Sodium (mmol/L) & $140(2)$ & $141(1)$ & 0.43 \\
\hline Urea (mmol/L) & $4.3(0.8)$ & $5.0(1.0)$ & 0.08 \\
\hline Osmolality (mOsm/kg) & $291(3.5)$ & 294 (2.9) & 0.01 \\
\hline Bicarbonate (mmol/L) & $27(1.5)$ & $27(1.8)$ & 0.38 \\
\hline Glucose (mmol/L) & $4.6(0.5)$ & $4.7(0.6)$ & 0.48 \\
\hline CRP (mg/L) & $0.6(0.6)$ & $0.5(0.4)$ & 0.48 \\
\hline Leukocytes $\left(1 \times 10^{9} / \mathrm{L}\right)$ & $4.8(0.4)$ & $5.5(1.0)$ & 0.03 \\
\hline Neutrophils $\left(1 \times 10^{9} / \mathrm{L}\right)$ & $2.3(0.5)$ & $2.8(0.6)$ & 0.03 \\
\hline Lymfocytes $\left(1 \times 10^{9} / \mathrm{L}\right)$ & $1.8(0.3)$ & $1.9(0.5)$ & 0.45 \\
\hline Monocytes $\left(1 \times 10^{9} / L\right)$ & $0.4(0.1)$ & $0.6(0.2)$ & 0.07 \\
\hline Eosinophils $\left(1 \times 10^{9} / \mathrm{L}\right)$ & $0.2(0.1)$ & $0.2(0.2)$ & 0.46 \\
\hline Basophils $\left(1 \times 10^{9} / \mathrm{L}\right)$ & $0.036(0.01)$ & $0.044(0.02)$ & 0.02 \\
\hline \multicolumn{4}{|l|}{ Twenty-four-hour urine } \\
\hline
\end{tabular}

surface proteins associated with monocyte adhesion, migration, and activation (CD11b, CD11c, CD18, CD29, CD49d, CD62L, CD32, CD45RA, CD64, CD86, TLR4, CD200R) were not significantly different between LSD and HSD (Supplemental Figure 2; supplemental material available online with this article; https://doi.org/10.1172/jci.insight.130508DS1). CD206 (i.e., mannose receptor C type 1, a marker for antiinflammatory macrophages) tended to be decreased after $\operatorname{HSD}(P=0.12$ and $P=0.10$ for the classical and intermediate subtype, respectively) (Figure 2C).

To assess whether HSD affects inflammatory responses, we challenged whole blood with LPS and measured inflammatory cytokine secretion. HSD resulted in an increased secretion of IL-6, but not of IL-8, IL-12, TNF, or MCP-1 (i.e., CCL2, the ligand of CCR2) upon LPS stimulation (Figure 3A). Accordingly, isolated monocytes showed increased IL-6 secretion $(P<0.001)$ when cultured in the presence of HS when compared with normal medium or medium containing urea as a tonicity control (Figure 3B). Having observed increased monocyte CCR2 expression after HSD, we also assessed the expression of its ligand MCP-1 (CCL2) in the presence of HS. Along with increased IL-6, monocytes tended to secrete more MCP-1 when cultured in the presence of HS in vitro $(P=0.09$, Figure $3 \mathrm{~B})$. Moreover, a HSD significantly increased blood plasma MCP-1 levels $(P<0.05$, Figure 3C), while we were unable to detect IL-6 in plasma of both LSD and HSD groups. Overall, no carryover effects could be found, meaning that diet order did not influence the observed effects. 
A

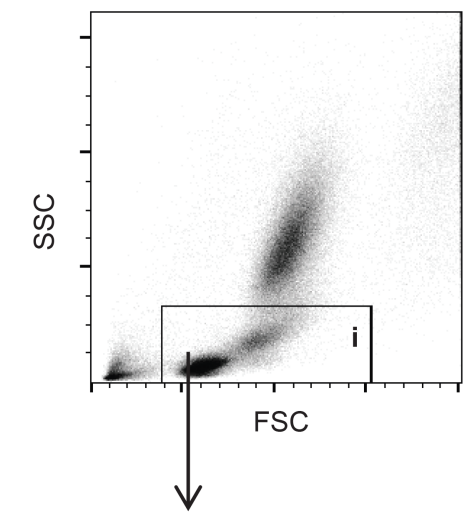

B

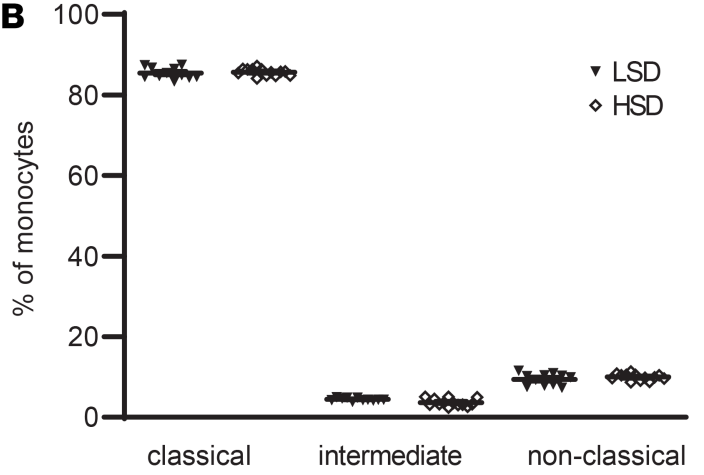

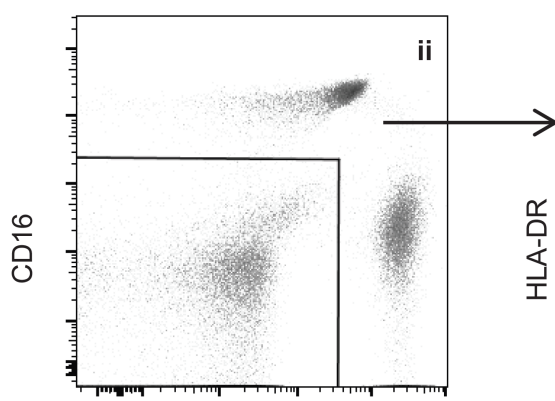

CD14

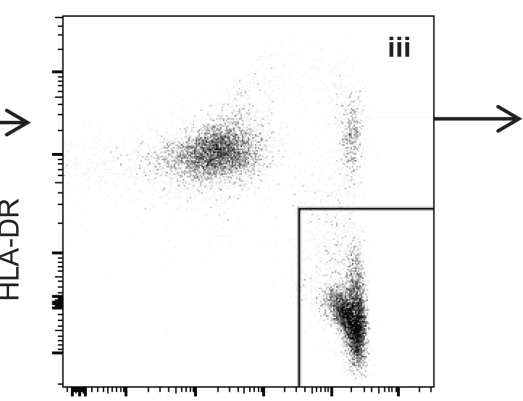

CD16

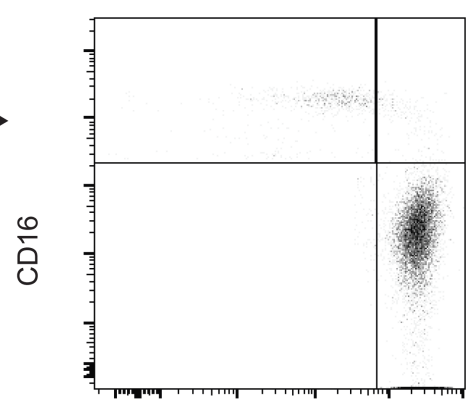

CD14
C

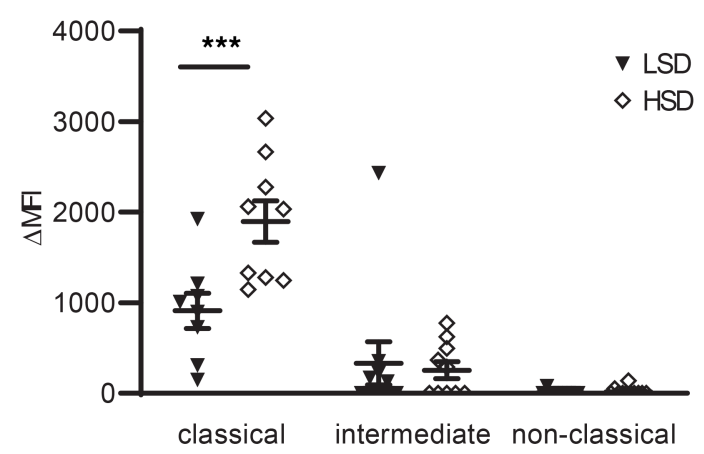

CCR2

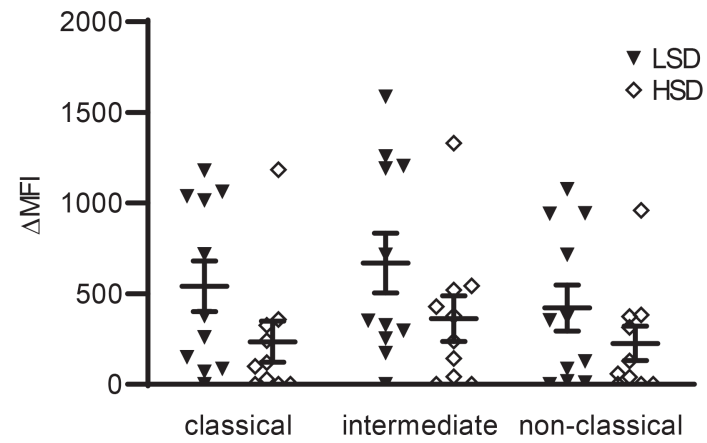

Figure 2. High-salt diet (HSD) induces a proinflammatory monocyte phenotype. (A) Gating of monocytes and monocyte subtypes. Debris, residual RBC, and granulocytes were first gated out on a FSC/SSC plot (gate i). Gate i was next displayed on a CD14/CD16 plot to select CD14+ and/or CD16 ${ }^{+}$cells (gate ii). Cells from gate ii were viewed on a CD16/HLA-DR plot to select monocytes (gate iii). This monocyte population was plotted again on a CD14/CD16 plot to gate

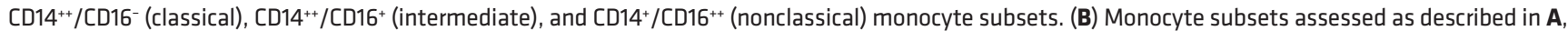
here expressed as a percentage of total monocytes (gate iii) after LSD and HSD. (C) The expression of various surface markers on the monocyte subsets was calculated as $\triangle \mathrm{MFI}=(\mathrm{MFI})_{\text {positive staining }}-(\mathrm{MFI})_{\text {isotype control }}$ CCR2 and CD2O6 are displayed here; other surface markers are depicted in Supplemental Figure 2. FSC, forward-scattered light. SSC, side-scattered light; LSD, low salt diet; MFI, median fluorescence intensity. Values represent mean \pm SEM of $n=11$ healthy male volunteers. Data tested using 2-way ANOVA. ${ }^{* *} P<0.001$.

HS increases monocyte infiltration. Since CCR2 plays a key role in monocyte chemotaxis (19), we investigated the effect of HS on transendothelial migration of monocytes and on tissue macrophage content. HS enhanced CCR2 expression on monocytes in vitro (Figure 4, A and B) and led to higher transendothelial migration (Figure 4C). In line with this, we found increased skin macrophage density after HSD (Figure $5, \mathrm{~A}$ and $\mathrm{B}$ ), as assessed in skin biopsies of 12 healthy males that participated in a previous dietary salt loading study (study II, detailed in Methods) (20). The mean (SD) age was $23( \pm 4)$ years and BMI was 22 $( \pm 2) \mathrm{kg}$. Dietary salt consumption at time of screening (as estimated based on urinary sodium excretion) averaged 9.5 grams/day (166 mmol sodium). Details on characteristics after LSD and HSD are provided in Supplemental Table 3. Notably, the increase in salt consumption from LSD to HSD was similar in both studies (study I, $+291.7[ \pm 119.3] \mathrm{mmol}$ sodium; study II, $+321.7[ \pm 110.0]$ mmol sodium; $P=0.54$ ). 
A

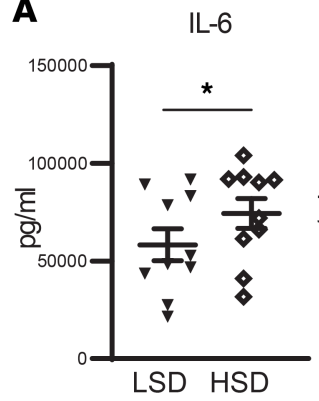

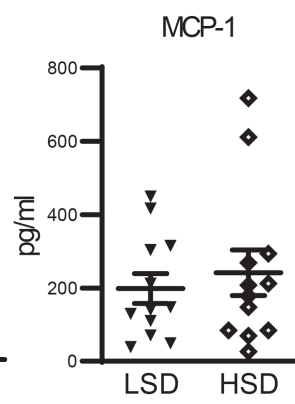
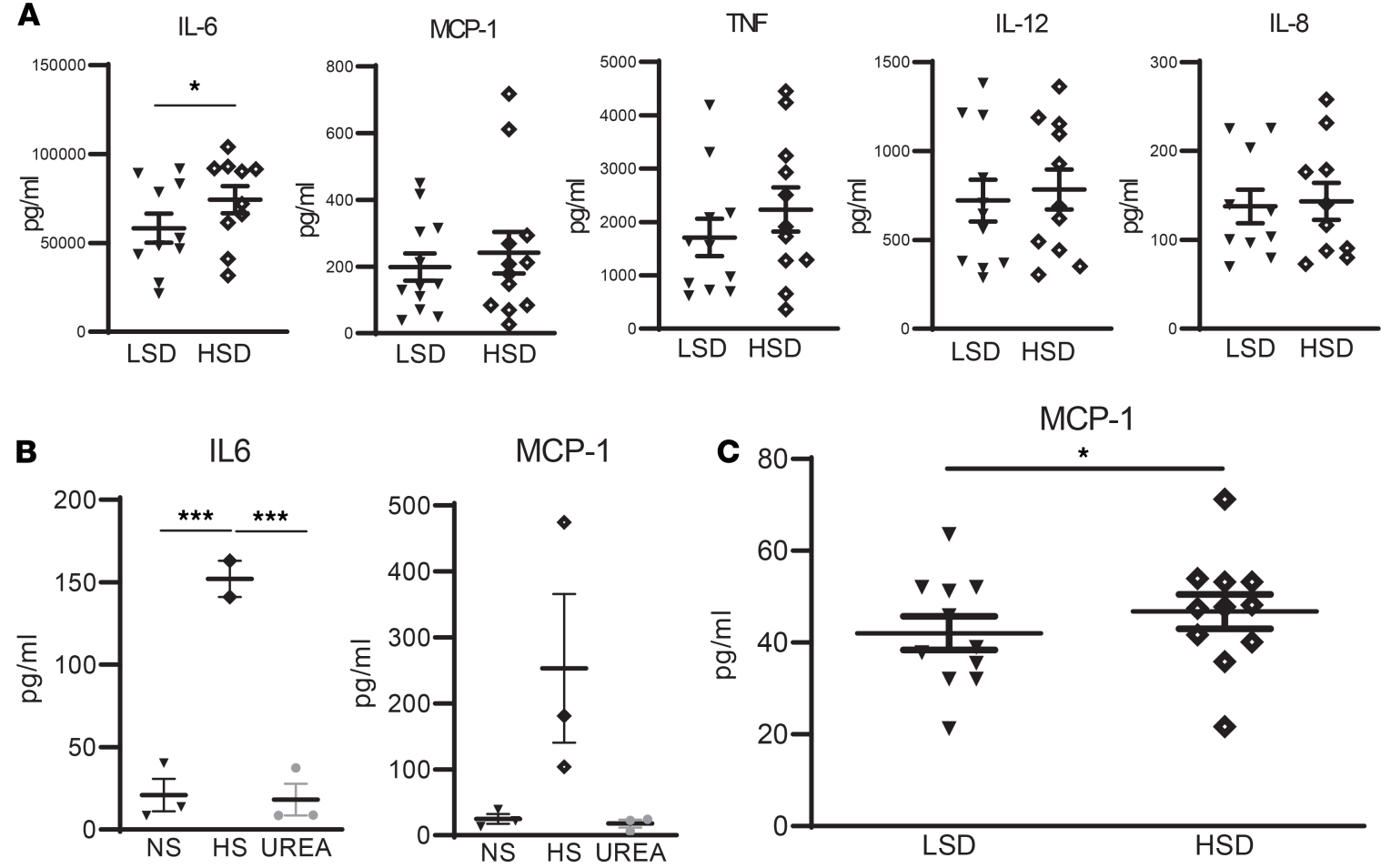

Figure 3. Effect of salt on cytokines. (A) Whole blood samples after LSD and HSD were challenged with LPS (24 h with $10 \mathrm{ng} / \mathrm{mL}$ ) and cytokines were quantified by ELISA. (B) Monocytes of independent healthy donors were stimulated for $24 \mathrm{~h}$ in RPMI $+10 \% \mathrm{FCS}+1 \%$ PenStrep in the presence of normal salt (NS), high salt (HS), or $80 \mathrm{mM}$ urea as tonicity control. Then, cytokine secretion was quantified by ELISA. (C) Plasma samples after LSD and HSD were analyzed for MCP- 1 and IL- 6 by ELISA. IL- 6 was below the detection threshold. For all analyses, ELISA was performed in accordance with the supplier's protocols (Invitrogen). NS, ([Na+] = $139 \mathrm{mM}) ; \mathrm{HS},\left(\left[\mathrm{Na}^{+}\right]=179 \mathrm{mM}\right)$. LSD, low-salt diet. HSD, high-salt diet. Values represent mean \pm SEM of $n=11$ healthy male volunteers (A and $\mathbf{C})$ and $n=3$ healthy male donors (B). Data tested using paired $t$ test (A and $\mathbf{C})$ or a 1-way ANOVA $(\mathbf{B}) .{ }^{*} P<0.05 ;{ }^{* * *} P<0.001$.

HS induces signs of proinflammatory activation in macrophages. To investigate whether HSD affects macrophage phenotype, we examined skin macrophage expression of HLA-DR as a proinflammatory marker and CD206 as an antiinflammatory macrophage marker. After HSD, macrophage HLA-DR expression was increased while CD206 expression was decreased (Figure 5, C-F). Also, given the effect of HSD on monocyte CCR2 and MCP-1 in the blood, we assessed the expression of both proteins in the skin and observed no differences between the 2 diets (Figure 5, G-J).

To investigate the effect of salt on macrophage responses, we cultured human macrophages differentiated from monocytes of healthy donors not participating in the dietary studies in HS medium. LPS-induced inflammatory macrophage activation in the presence of HS enhanced the release of IL-6, TNF, and IL-10 and had no effect on IL-12 secretion in comparison with macrophages that were stimulated in normal salt (NS) conditions or in the presence of urea as a tonicity control (Figure 6, A and B). Macrophages that were also differentiated in the presence of HS showed an even more prominent inflammatory cytokine release upon LPS exposure (Supplemental Figure 3). Since salt was previously shown to blunt M2 macrophage polarization in mice (14), we next investigated IL-4-induced macrophage responses in human macrophages. In vitro, HS did not reduce the activation of IL-4-stimulated human macrophages, as demonstrated by similar CD206 and CD200R surface expression (Figure 6, C and D, and Supplemental Figure 4). Strikingly, the IL-4-induced expression of several marker genes including FCER2, CCL24, CLEC10A, and SOCS1 were significantly increased in HS conditions (Figure 6E). Factually, of all M(IL-4) signature genes tested, CD200R was the only one that was suppressed by HS (Figure 6E).

\section{Discussion}

In this study, we have demonstrated that HS increases CCR2 expression on monocytes in vitro and in vivo, coinciding with increased plasma MCP-1, increased salt-induced transendothelial migration of monocytes, and increased skin macrophage content after HSD. Macrophages demonstrate signs of an increased proinflammatory phenotype after salt exposure, as assessed by increased HLA-DR expression and decreased 

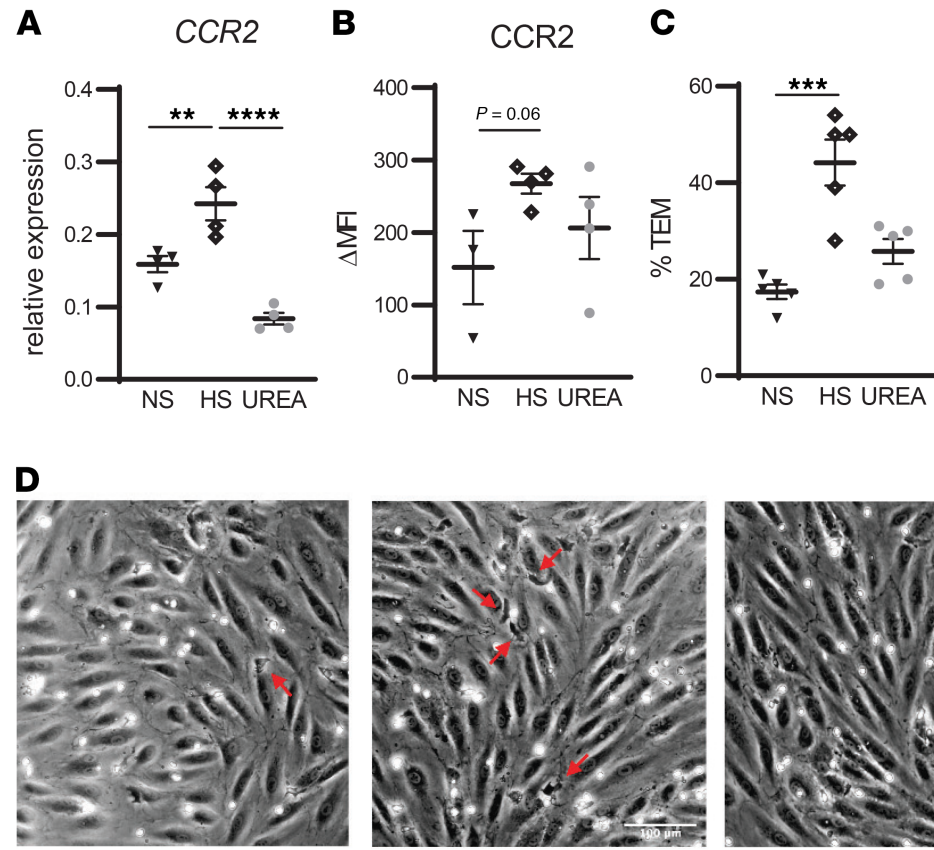

NS

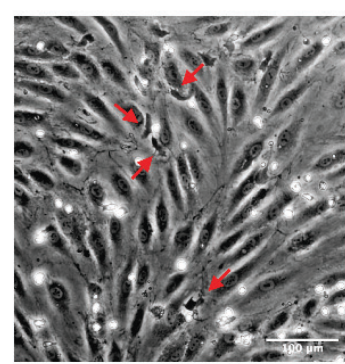

HS

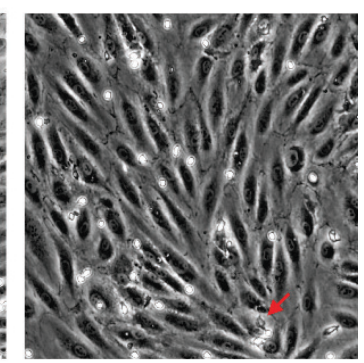

UREA

Figure 4. High salt increases CCR2 expression and transendothelial migration of monocytes in vitro. (A and B) Monocytes of independent healthy donors were stimulated for $24 \mathrm{~h}$ in RPMI $+10 \% \mathrm{FCS}+1 \%$ PenStrep in the presence of normal salt (NS), high salt (HS), or $80 \mathrm{mM}$ urea as tonicity control. Then, CCR2 gene expression was assessed with qPCR (A) and protein expression with flow cytometry (B). (C and D) After 24-h incubation in NS, HS, or urea, monocytes were added to the monolayer of cultured human arterial endothelial cells. Transmigrated monocytes (red arrows) were distinguished from adhered monocytes by their transitions from bright to black morphology. NS, ([Na+ $]=139 \mathrm{mM}) ; \mathrm{HS}$, $\left(\left[\mathrm{Na}^{+}\right]=179 \mathrm{mM}\right)$. Values represent mean \pm SEM of $n=4(\mathbf{A}$ and $\mathbf{B})$ and $n=5(\mathbf{C})$ healthy donors. Data tested using 1-way ANOVA. ${ }^{* *} P<0.01 ;{ }^{* *} P<0.001 ;{ }^{* * *} P<0.0001$.

CD206 expression on skin macrophages after HSD, and as represented by boosted LPS-induced cytokine secretion of IL-6, TNF, and IL-10 in vitro.

We did not observe any HSD-induced increase of the intermediate monocyte subset, which was found in an earlier study, or an increase in any other subset (12). We did find increased CCR2 expression on the classical monocyte subset after HSD and on isolated monocytes exposed to HS in vitro. In contrast to earlier findings (16), CCR2 expression on macrophages exposed to HS in vitro did not increase in our study (data not shown), nor did HSD increase skin macrophage CCR2 expression. This is not totally unexpected, given the fact that CCR2 is a marker mostly present on monocytes and is known to be downregulated when maturation toward macrophages occurs $(21,22)$. CCR2 is a chemokine receptor that mediates monocyte infiltration in inflammatory diseases (6). In line with this, we observed increased salt-induced transendothelial migration of monocytes in vitro, as well as increased skin macrophage content after HSD. Also, we found increased MCP-1 secretion by monocytes incubated with HS and increased plasma MCP-1 after HSD, the latter in concordance with earlier observations (23). The essential role of the MCP-1/CCR2 axis in monocyte migration has been shown for a wide range of tissues (including skin, vessel wall, lungs, the central nervous system, and peritoneum, amongst others; refs. 24-30) by numerous animal studies using MCP-1 or CCR2 KOs. More specifically, Sakata et al. showed that HSD-induced macrophage infiltration into heart and peritoneal wall tissue was reduced in CCR2-KO mice compared with WT (31). Although together with our data, these observations point toward a role for CCR2-mediated transendothelial migration after salt exposure, future studies need to scrutinize the exact mechanistic pathways. Also, other factors may play a role, like salt-induced alterations of the endothelium — for example, by increased VCAM-1and ICAM-1-dependent monocyte-endothelium adhesion $(32,33)$. Additionally, tissue sodium storage may be contributing to monocyte influx, since tissue sodium content increases with HSD $(10,34)$ and seems to act as a chemotactic stimulus for macrophages (35). Furthermore, complex interactions between different types of immune cells may take place. For example, there is increasing evidence that 
A

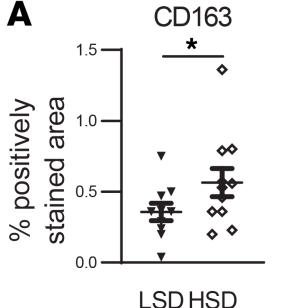

C

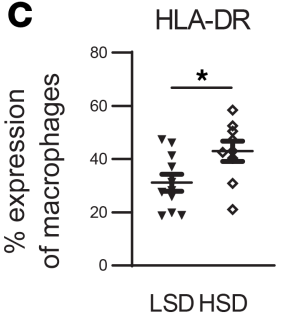

$\mathbf{E}$
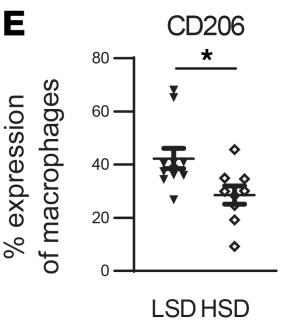

G

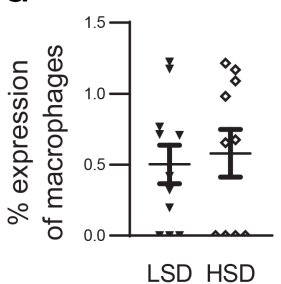

I

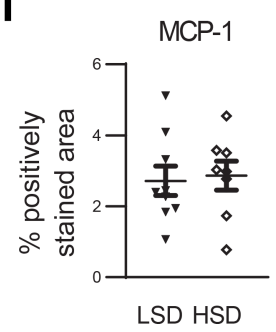

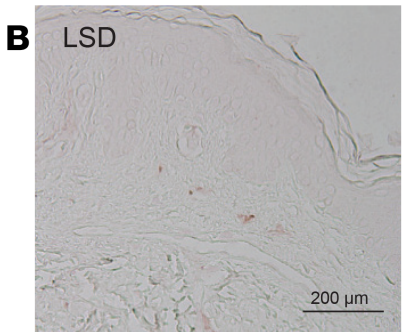

D LSD
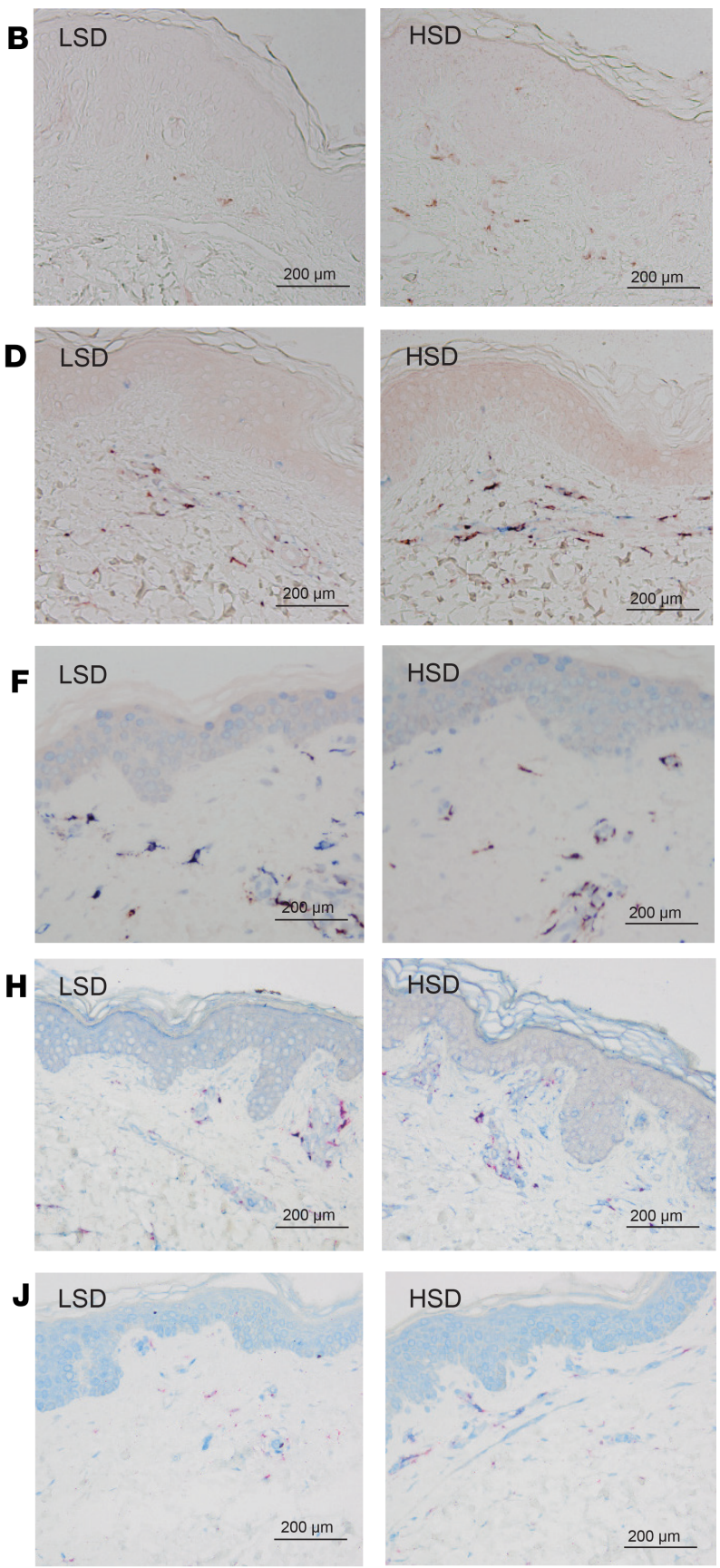

Figure 5. HSD increases skin macrophage content with signs of a more proinflammatory and less antiinflammatory phenotype. (A and B) To assess total skin macrophage content, sections were stained with anti-CD163 (red). Quantification of total macrophage content was expressed as the percentage of the total dermal area of the section that was positively stained for anti-CD163 (A). Anti-CD163 was favored above anti-CD68, since our pilot experiments showed that CD163 proved to be a more consistent macrophage marker than CD68 (Supplemental Figure 1), which is in line with the observations of 2 other research groups (51, 52). (C-H) To assess expression of proinflammatory and antiinflammatory macrophage markers, sections were stained with anti-CD163 (red) and either anti-HLA-DR (blue) (C and D), anti-CD206 (blue) (E and F), or anti-CCR2 (blue) (G and H). Since HLA-DR, CD206, and CCR2 can be expressed by other cells than macrophages, we only took into account the positively stained area of the concerning marker that was also positive for the macrophage marker anti-CD163. Quantification of the total expression of the several markers by macrophages was expressed as the percentage of positively stained area for anti-CD163 that was also positively stained for the concerning marker. (I and J) To assess skin expression of MCP-1, section were stained with anti-MCP-1 (blue) and anti-CD163 (red). Quantification of skin expression of MCP-1 was expressed as the total dermal area of the section that was positively stained for MCP-1 (I). LSD, low-salt diet. HSD, high-salt diet. Scale bar: $200 \mu \mathrm{m}$. Values represent mean \pm SEM of $n=9-11$ healthy male volunteers. Data are tested using a paired $t$ test. ${ }^{*} P<0.05$. 
A

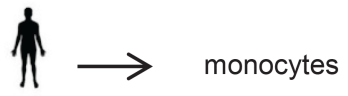

$\left[\mathrm{Na}^{+}\right]=139 \mathrm{mM} \quad \begin{array}{ll}7 \mathrm{~d} \\ \mathrm{M}-\mathrm{CSF}\end{array}$

$\mathrm{M}(0)$

$24 \mathrm{~h}$

+/- LPS

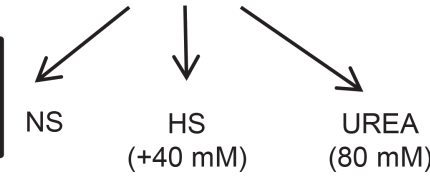

C

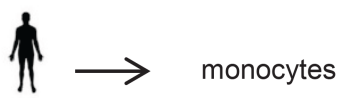

$\left[\mathrm{Na}^{+}\right]=139 \mathrm{mM} \quad \begin{array}{ll}7 \mathrm{~d} \\ \mathrm{M}-\mathrm{CSF}\end{array}$

$24 \mathrm{~h}$

$+/-$ IL4
$M(0)$

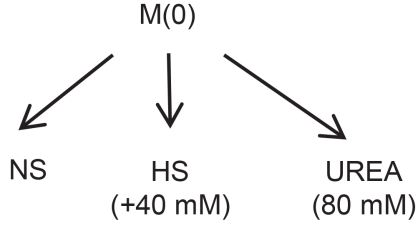

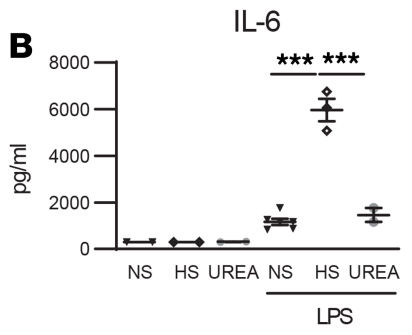

IL-12
IL-10

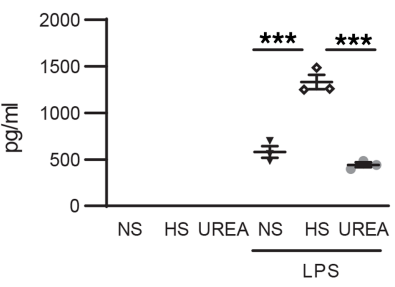

TNF

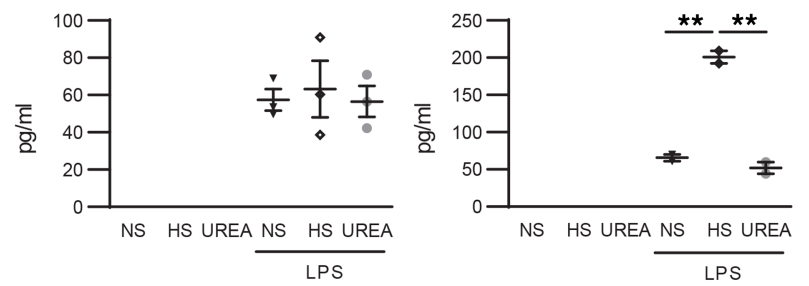

D

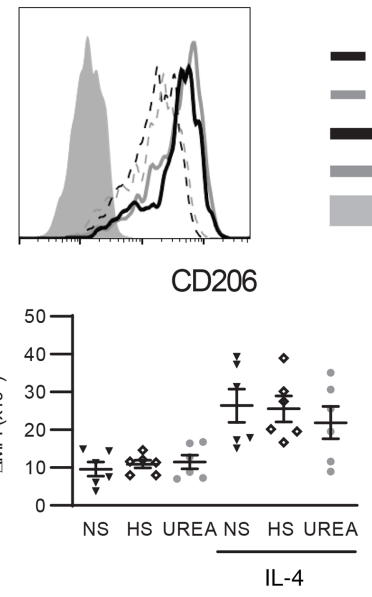

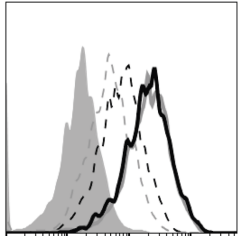

CD200R

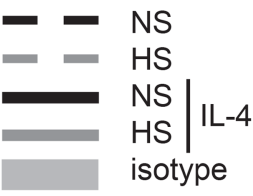

isotype

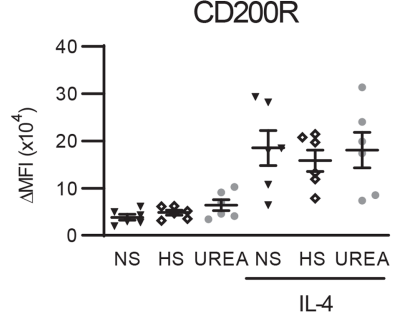

E

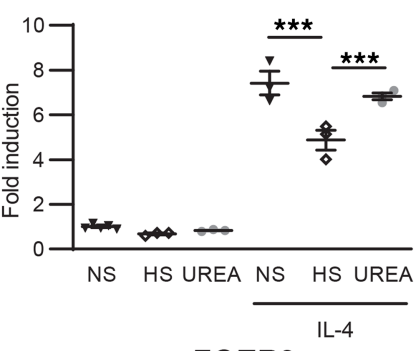

FCER2

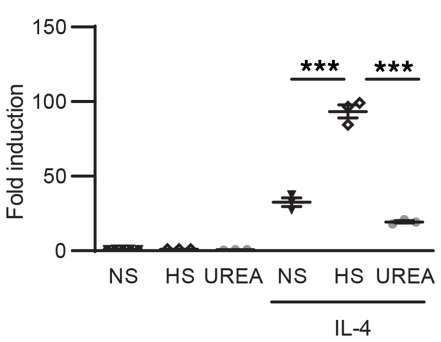

CD206

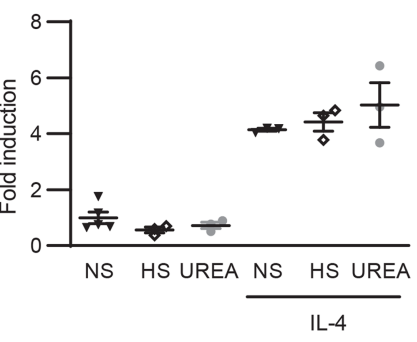

CCL24

TGM2

CCL22

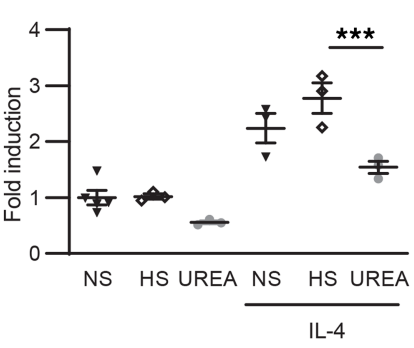

CLEC10A
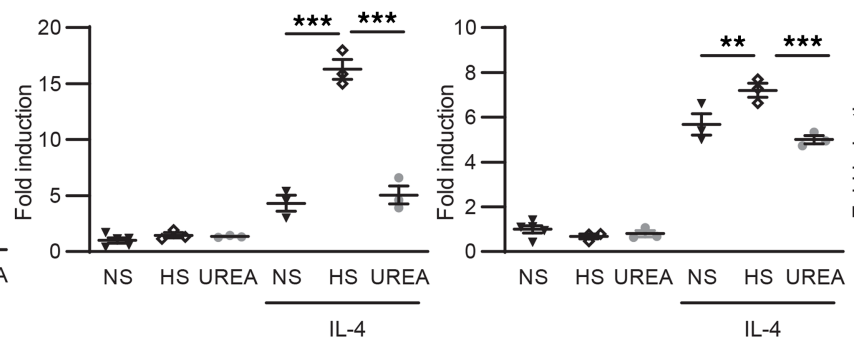

Figure 6. High salt (HS) boosts LPS-induced cytokine secretion in macrophages in vitro but does not affect the activation of M(IL-4). (A) Schematic overview of in vitro LPS stimulation of macrophages. Monocytes of healthy volunteers were cultured in the presence of M-CSF for 7 days to differentiate into mature macrophages and stimulated for $24 \mathrm{~h}$ with LPS in the presence of normal salt (NS) concentrations present in IMDM medium (NS; $\left.\left[\mathrm{Na}^{+}\right]=139 \mathrm{mM}\right), \mathrm{HS}\left(\left[\mathrm{Na}^{+}\right]=179 \mathrm{mM}\right.$; i.e., IMDM medium supplemented with an additional $\left.40 \mathrm{mM} \mathrm{NaCl}\right)$, or 80 mM urea as tonicity control. (B) IL-6, IL-10, IL-12, and TNF were quantified by ELISA. (C) Schematic overview of in vitro IL-4 stimulation of macrophages. (D) Differentially treated macrophages were stained with antibodies against the M2 surface markers CD206 and CD200R, or isotype control, followed by flow cytometric analysis. 
Representative histogram graphs and corresponding surface expression quantifications $\left(\Delta \mathrm{MFI}=[\mathrm{MFI}]_{\text {positive staining }}-[\mathrm{MFI}]_{\text {isotype staining }}\right)_{\text {are }}$ presented. $(\mathbf{E})$ Gene expression of IL-4-induced M2 marker genes. The fold inductions of indicated marker genes are shown relative to the expression in untreated macrophages (= 1). M-CSF, macrophage CSF; NS, ([Na+] = $139 \mathrm{mM}) ; \mathrm{HS},\left(\left[\mathrm{Na}^{+}\right]=179 \mathrm{mM}\right)$; MFI, median fluorescence intensity. Values represent mean \pm SEM of at least $n=3$ healthy male donors. $n=3$ (1 outlier excluded for TNF for $\mathbf{B}, n=6$ for $\mathbf{D}, n=3$ for $\mathbf{E}$ ). Data tested using 1 -way ANOVA. ${ }^{*} P<0.05$; ${ }^{*} P<0.01 ;{ }^{* *} P<0.001$

neutrophil activation and influx precede and are essential for monocyte influx $(27,36)$. In our study, we observed an HSD-induced increase in neutrophils and basophils in the blood, along with the trend toward monocyte increase. Mechanisms and relevance for these cell types need to be studied further.

In line with previous findings in mice, our data demonstrate that HS augments LPS-induced proinflammatory macrophage responses in humans (15). However, our data highlight clear differences between human and mouse macrophages with regard to IL-4-induced macrophage activation in the presence of increased salt in vitro. Whereas alternative macrophage activation is blunted by HS in mice (14), IL-4induced responses in human macrophage cultures appear to be unaffected or are even increased in high-salt conditions. The observed responses in skin macrophages after HSD are more in line with animal studies, showing increased skin macrophage content after HSD (10), with signs of increased proinflammatory activation and decreased antiinflammatory activation $(6,10,11)$. Additionally, it validates our in vitro data that reveal increased proinflammatory activation of macrophages subjected to a high-salt medium.

Interestingly, proinflammatory priming of both monocytes and macrophages, as well as CCR2, have been shown to play causal roles in hypertension development $(11,37-39)$. In mouse models, a reduction in monocytes by selective ablation attenuated angiotensin-II-induced blood pressure increases, and adoptive transfer of proinflammatory monocytes reestablished the blood pressure increase - adoptive transfer of monocytes without a proinflammatory phenotype did not have any effect (38). Anti-CCR2 has been shown to prevent hypertension development in rats and mice subjected to a $\operatorname{HSD}(11,37)$, which is in line with our observations that the HSD-induced inflammatory priming and CCR2 increase coincided with a $5 \mathrm{mmHg}$ systolic blood pressure increase. Our study, however, lacked power to perform reliable subgroup analyses to assess the relation between CCR2 and individual salt sensitivity of blood pressure.

Furthermore, proinflammatory activation and CCR 2 have been implicated in acute and chronic kidney disease $(9,40,41)$ and cardiovascular disease $(6,42,43)$, underlining the relevance of our findings for kidney and cardiovascular patients. Patients with chronic kidney disease show increased arterial inflammation and increased proinflammatory phenotype of monocytes, as displayed by increased CCR7 and CCR 2 expression (9). Reduction of CCR2-expressing monocytes by inhibition of MCP-1 has been shown to reduce proinflammatory priming of renal macrophages and reduced albuminuria in diabetic nephropathy (41). With regard to cardiovascular disease, proinflammatory monocytes, and the MCP-1/CCR2 axis in particular, have been implicated in the development and progression of pathologies like atherosclerosis and myocardial infarction (reviewed in ref. 6). Among others, anti-CCR2 and the absence of CCR2 reduce atherosclerosis in mice prone for atherosclerosis development $(42,43)$. Taken together, our data may provide a link between salt and its deleterious outcomes, which is relevant for the healthy population, as well as for patients with cardiovascular and kidney disease. The exact mechanisms by which proinflammatory priming of monocytes and macrophages contribute to development or progression of hypertension, kidney disease, and cardiovascular disease need to be further scrutinized but may involve vascular endothelial dysfunction and reduced vascular superoxide formation, among others, as suggested by previous animal studies $(38,44)$. Of note, although HS may induce proinflammatory effects on monocytes and macrophages specifically and induce a plasma MCP-1 increase, other circulatory proinflammatory markers may decrease, as shown by a study in hypertensive subjects that demonstrated decreased TNF and procalcitonin with high salt intake (45).

Strong points of our study are the randomized dietary salt loading protocol that adheres to recent recommendations (46) and the translational character. Our in vitro findings underline that the observed effects after dietary salt loading are (at least partly) due to salt itself and are not the results of accompanying HSD-induced effects on, for example, hemodynamics or volume status. However, some limitations have to be taken into consideration. The use of a powerful stimulant for the in vitro macrophage experiments may have clouded more subtle pathways activated by salt. Also, since our in vitro macrophage experiments were aimed at validating previous findings in mice, we tried to replicate earlier experimental settings and cultured the cells in medium containing 10\% FCS. As such, we ensured that possible observed differences are due to differences between mice and humans and not due to experimental differences. 
However, it should be noted that the use of distinct serum (FCS versus human) results in distinct macrophage types, and therefore serum-specific effects cannot be excluded (47). Additionally, the observed slight increase in IL-6 release after HSD (whole blood stimulation with LPS) and after HS exposure in vitro (on supernatants from isolated monocytes) might - although statistically significant — not be clinically relevant, since no IL-6 increase was found after HSD in plasma. While monocytes are expected to be the main producers of these inflammatory mediators, our data do not rule out the possibility that other cells contribute to increased levels of IL-6 upon whole blood LPS challenge. In general, the role of this cytokine needs to be further established, since IL-6 has both atherogenic and atheroprotective roles and since studies with IL-6 blocking antibodies are inconclusive (48). Furthermore, the modest sample size limited us from exploration of observed monocyte or macrophage changes with individual salt sensitivity (by, for example, subgroup analyses on blood pressure). For this, future and larger-scale studies are needed. Lastly, although we are - to our knowledge - the first to demonstrate detailed effects of dietary salt on the MCP-1/CCR2 axis in the mononuclear phagocyte system in humans, further studies are needed to provide insight into the exact mechanistic pathways and to establish whether the observed changes are either pathophysiological or physiological.

In conclusion, we demonstrate that HS promotes an inflammatory monocyte phenotype characterized by strongly enhanced expression of CCR2, together with increased plasma MCP-1, increased transendothelial migration of monocytes, and increased human tissue macrophage content. Macrophages demonstrate signs of increased proinflammatory priming after salt exposure. As such, this study might bring us one step closer to understanding the deleterious effects of high salt intake.

\section{Methods}

Study design and procedures. We carried out a prospective randomized crossover dietary salt intervention study (study I) in male, nonsmoking healthy subjects between 18 and 40 years of age. We excluded overweight subjects $(\mathrm{BMI}>30)$ and subjects with a blood pressure above 140/90 $\mathrm{mmHg}$. All subjects adhered to HSD (aim of $>12 \mathrm{~g} \mathrm{NaCl} /$ day) and LSD (aim of $<3 \mathrm{~g} \mathrm{NaCl}$ /day) for 14 days, in randomized order without a wash-out period between, and refrained from performing heavy exercise, drinking alcohol, or smoking. For the skin macrophage analyses, material from a previous dietary salt intervention study (study II) was used (20). This study set-up was identical to study I; the only difference was that the diets lasted for 7 days. Diets were pursued with the help of a dietary list, which advised to resemble the normal diet of the individual as much as possible (e.g., by adding extra salt instead of changing the whole dietary pattern). On days 7, 11, and 15 of both diets (days 3, 5, and 8 for study II), sodium and creatinine in 24-hour urine samples were assessed to check for compliance (the samples of day 15 [day 8 for study II] were used for analyses). On day 15, after an overnight fast, blood samples were obtained for cytometry analysis. Brachial blood pressure was measured with a semiautomatic wrist blood pressure device (Omron) in a sitting position after at least 5 minutes of rest in a quiet room. Blood pressure measurements were performed at least 3 times at the nondominant arm with a 10 -second interval. The mean of the last 2 consecutive measurements with less than $5 \mathrm{mmHg}$ differences were taken to represent brachial blood pressure.

Randomization and blinding. Randomization was done in blocks of 4 via sealed envelopes by the study investigators. Diet status was not masked for the study subjects or investigators during follow-up. Blinding was ensured during flow cytometry and IHC and subsequent data analysis.

Flow cytometry. Blood was collected in K3 EDTA BD Vacutainer (BD Biosciences) tubes by peripheral venipuncture. After lysis of RBC with RBC lysis buffer (eBioscience), cells were washed with PBS and subsequently incubated with fluorescent antibodies (Supplemental Table 1) for 20 minutes at room temperature in the dark. I.v. immunoglobulin (IVIG, Sanquin) was used to block nonspecific binding. After washing, data were acquired with a Beckman Coulter Cytoflex and analyzed with FlowJo, using eBioscience OneComp eBeads for compensation. Monocytes were gated as described previously (49). Debris, residual RBC, and granulocytes were first gated out on a forward scatter/side scatter (FSC/SSC) plot (gate i; Figure 2). Gate i was next displayed on a CD14/CD16 plot to select $\mathrm{CD}_{14}{ }^{+}$and/or CD16 ${ }^{+}$cells (gate ii). Cells from gate ii were viewed on a CD16/HLA-DR plot to select monocytes (gate iii). This monocyte population was plotted again on a CD14/CD16 plot to gate CD14 ${ }^{++} / \mathrm{CD} 16^{-}$(classical), CD14 ${ }^{++} / \mathrm{CD} 16^{+}$(intermediate), and $\mathrm{CD} 4^{+} / \mathrm{CD} 6^{++}$(nonclassical) monocyte subsets. The expression of various surface markers on these monocyte subsets was calculated as $\Delta$ median fluorescence intensity $(\triangle \mathrm{MFI})=(\mathrm{MFI})$ $-(\mathrm{MFI})$ 
Whole blood challenge and ELISA. Fresh blood was diluted 1/5 in RPMI1640 medium, plated in 6-well plates, and treated for 24 hours with $10 \mathrm{ng} / \mathrm{mL}$ LPS. Next, medium was collected, centrifuged at 20,000 g for 5 minutes at room temperature and stored at $-80^{\circ} \mathrm{C}$ until subsequent analysis. MCP-1, IL-6, IL-8, IL-10, IL-12(p70), and TNF were quantified in whole blood (after LPS challenge), supernatants from isolated monocytes, and plasma by ELISA in accordance to the suppliers' protocols (Invitrogen).

Monocyte isolation for in vitro experiments. Peripheral blood mononuclear cells were obtained from whole blood samples from male healthy donors who did not participate in the salt intervention through density centrifugation using Lymphoprep (density [d] = 1.077). Blood was diluted in a 1:1 ratio with PBS/2 mM EDTA and subsequently added to a layer of Ficoll-Paque PLUS (Axis-Shield). Next, cells were centrifuged for 20 minutes at $600 \mathrm{~g}$ at room temperature with slow acceleration and no brake. The peripheral blood mononuclear cell fraction was collected and washed twice with PBS/2 mM EDTA. Next, cells were counted using a CASY counter (CASY TT; Roche Innovatis). Subsequently, monocytes were isolated using human CD14 magnetic beads and MACS cell separation columns according to the manufacturer's protocol (Miltenyi Biotec). In short, cells were resuspended in MACS buffer (0.5\% BSA [Sigma-Aldrich] in PBS/2 mM EDTA). Next, CD14 MicroBeads (Miltenyi Biotec) were added and incubated for 30 minutes at $4^{\circ} \mathrm{C}$. After incubation, the cells were washed with MACS buffer and resuspended in MACS buffer for CD14 bead isolation using MACS separation columns (Miltenyi Biotec). After isolation, cells were counted by means of a CASY counter.

In vitro human macrophage and monocyte cultures. Monocytes were incubated in RPMI $+10 \% \mathrm{FCS}+1 \%$ PenStrep (Thermo Fisher Scientific) for 24 hours with either NS concentrations $\left(\left[\mathrm{Na}^{+}\right]=139 \mathrm{mM}\right)$, HS $\left(\left[\mathrm{Na}^{+}\right]=179 \mathrm{mM}\right.$; i.e. medium supplemented with an additional $\left.40 \mathrm{mM} \mathrm{NaCl}\right)$, or $80 \mathrm{mM}$ urea as tonicity control. After this, gene expression was analyzed, as detailed below. For the macrophage experiments, cells were cultured in IMDM (Invitrogen) with $25 \mathrm{mM}$ HEPES and $2 \mathrm{mM}$ L-glutamine, supplemented with $10 \%$ FCS, penicillin $(100 \mathrm{U} / \mathrm{mL})$, streptomycin $(100 \mu \mathrm{g} / \mathrm{mL})$ (Thermo Fisher Scientific), and $50 \mathrm{ng} / \mathrm{mL}$ human M-CSF (Miltenyi Biotec) at $1 \times 10^{6}$ cells $/ \mathrm{mL}$ for 7 days to differentiate them into mature macrophages. Next, cells were stimulated 24 hours with $10 \mathrm{ng} / \mathrm{mL}$ LPS (Sigma-Aldrich) or $50 \mathrm{ng} / \mathrm{mL}$ IL-4 (Peprotech) in the presence of NS concentrations present in IMDM medium (NS; $\left.\left[\mathrm{Na}^{+}\right]=139 \mathrm{mM}\right), \mathrm{HS}\left(\left[\mathrm{Na}^{+}\right]=179 \mathrm{mM}\right.$; i.e., IMDM medium supplemented with an additional $40 \mathrm{mM} \mathrm{NaCl}$ ), or $80 \mathrm{mM}$ urea as tonicity control. Alternatively, cells were differentiated and stimulated in NS or HS during the entire culture period. LPS- and IL-4-induced macrophage activation was assessed by flow cytometry (Supplemental Table 1 for antibodies), ELISA, and gene expression analysis as detailed below. Fixable Viability Dye eFluor780 (eBioscience) was used to eliminate dead cells when performing flow cytometry on macrophages stimulated in vitro.

Gene expression analysis. RNA was isolated with High Pure RNA Isolation kits (Roche Diagnostics), cDNA was synthesized with iScript (Bio-Rad), and quantitative PCR (qPCR) was performed using Sybr Green Fast mix (Applied Biosytems) on a ViiA7 (Applied Biosystems). Housekeeping genes GNB2L1 and HPRT1 were used for normalization. Primer sequences are given in Supplemental Table 2.

Transendothelial monocyte migration assay. Primary human arterial endothelial cells (Lonza) were cultured to confluence. Subsequently, cells were stimulated overnight with $10 \mathrm{ng} / \mathrm{mL}$ TNF (Peprotech). Prior to adding monocytes to the monolayer (which were isolated as described above and incubated in RPMI $+10 \%$ FCS $+1 \%$ PenStrep for 24 hours with either NS concentrations $\left(\left[\mathrm{Na}^{+}\right]=139 \mathrm{mM}\right), \mathrm{HS}\left(\left[\mathrm{Na}^{+}\right]=\right.$ $179 \mathrm{mM}$; i.e., medium supplemented with an additional $40 \mathrm{mM} \mathrm{NaCl}$ ), or $80 \mathrm{mM}$ urea as tonicity control), medium was changed for EGM-2 culture medium (Lonza) to remove TNF. Stimulated monocytes were added in a concentration of $1 \times 10^{6} \mathrm{cells} / \mathrm{mL}$ for 30 minutes at $37^{\circ} \mathrm{C}, 5 \% \mathrm{CO}_{2}$, and then fixed with $3.7 \%$ formaldehyde (Sigma-Aldrich). Images were recorded using a Leica DM8i Live-Cell miscroscope (Plan-apochromat 10×/0.25 Phaco 1; Leica). Adherent cells were visualized by phase-contrast microscopy, where - due to the imaging modality - adherent cells have a bright morphology (white, lying on top of the endothelial layer) and transmigrated cells show a dark phase morphology (black) (Figure 4D). At least 3 images were quantified per condition. Quantification was performed with ImageJ2 (NIH) software (50).

Skin biopsy and IHC. After each diet, a skin biopsy of the medial side of the upper arm was obtained. After local anesthetics with $0.5 \mathrm{~mL}$ lidocaine $1 \%$ (Fresenius Kabi), a punch biopsy of $3 \mathrm{~mm}$ in diameter was performed. The tissue was fixed in formalin and embedded in paraffin. For IHC, 4- $\mu \mathrm{m}$-thick sections were cut and mounted on glass slides. After deparaffinization and rehydration, endogenous peroxidase activity of the samples that were developed by an HRP-conjugated secondary antibody was blocked with methanol (VWR Chemicals) plus $0.3 \%$ peroxide for 15 minutes at room temperature. Heat-induced epitope retrieval of rehydrated sections was performed using $10 \mathrm{mM}$ Tris-EDTA (consisting of UltraPure Tris [Invitrogen] and Titriplex III [Merck]), 
$\mathrm{pH} 9.0$, for 20 minutes at $98^{\circ} \mathrm{C}$, followed by a cool-down period of 30 minutes at room temperature. After 3 times washing with PBS, Ultra V Block (Thermo Fisher Scientific/Labvision) was applied for 30 minutes at room temperature. Subsequently, the primary antibodies (mouse IgG1 anti-HLA-DR, 1:200, M0746, clone TAL.1B5, Dako; rabbit IgG anti-CD206, 1:200, ab64693, Abcam; mouse IgG2b $\lambda$ anti-CCR2, 1:200, NBP235334, clone 3G7, Novus Biologicals; and mouse IgG1 anti-MCP-1, 1:200, NBP2-22115, clone 2D8, Novus Biologicals) were applied and incubated 1 hour at room temperature (for anti-CCR2 overnight at $4^{\circ} \mathrm{C}$ ). Antibody binding was detected with goat IgG anti-mouse IgG (DPVM110AP, ImmunoLogic) or goat IgG anti-rabbit IgG (VWRKDPVR110AP, ImmunoLogic) AP-conjugated polymer (Diagnostics BioSystems), as appropriate, and AP activity was visualized using PermaBlue Plus/AP (Diagnostic BioSystems). To detect colocalization of the primary antibodies with macrophages, a secondary staining step was implemented. Heat-induced epitope retrieval was performed using $10 \mathrm{mM}$ Tris-EDTA, $\mathrm{pH} 9.0$, for 20 minutes at $98^{\circ} \mathrm{C}$ to remove the first set of immunoreagents and leaving the PermaBlue precipitate unchanged. After a cool-down period of 30 minutes at room temperature and 3 times washing with PBS or TBST, slides were incubated with mouse IgG1 anti-CD163 (1:200, 10D6, NeoMarkers) for 1 hour at room temperature. Antibody binding was detected with goat IgG antimouse IgG HRP-conjugated polymer (VWRKDPVM110HRP, ImmunoLogic) or goat IgG anti-mouse IgG AP-conjugated polymer (DPVM110AP, ImmunoLogic). HRP activity was visualized with NovaRed (Vector Laboratories), and AP activity was visualized with PermaRed Plus/AP (Diagnostic BioSystems). Sections were mounted with VectaMount (Vector Laboratories), without a nuclear counterstain. Brightfield imaging was performed using an Olympus BX51 microscope with a UPlanF1 20×/0.5 objective and DP70 camera (Olympus). Images were analyzed with ImageJ2 software (50). Macrophage density was expressed as the percentage of the total dermal surface area that was positively stained for anti-CD163. Anti-CD163 was favored above anti-CD68, since our pilot experiments showed that CD163 proved to be a more consistent macrophage marker than CD68 (Supplemental Figure 1), which is in line with the observations of 2 other research groups $(51,52)$. Next, macrophage expression of HLA-DR and CD206 was quantified to determine the polarization of macrophages toward a more proinflammatory (M1) phenotype (HLA-DR) or toward a more antiinflammatory (M2) phenotype (CD206). Also, macrophage expression of CCR2 and tissue expression of MCP-1 was assessed. To exclude the expression of HLA-DR, CD206, and CCR2 on cells other than macrophages in the measurements, only the area that was positively stained for both CD163 and one of these markers was taken into account and expressed as a percentage of total positively stained CD163 area. All analyses were performed in a blinded fashion and semiautomatically.

Statistics. Continuous variables are reported as mean \pm SEM or SD, as reported. Outliers were detected by means of the Grubb's test and were excluded from the analysis. Differences between LSD and HSD were calculated by a 2-tailed paired $t$ test or a Wilcoxon as appropriate. For the monocyte experiments, data were tested using 2-way ANOVA with factors monocyte subtype and diet, followed by Bonferroni's post hoc comparisons tests. For the in vitro monocyte and macrophage experiments, data were tested using 1-way ANOVA followed by Bonferroni's post hoc comparisons tests. To test for carryover effects, the changes between LSD and HSD of the group in which LSD was pursued before HSD (LSD-HSD group) were compared with the changes between LSD and HSD of the HSD-LSD group, with a Mann-Whitney $U$ test. Analyses were conducted with SPSS (version 22.0, SPSS Inc.) and GraphPad (version 7.00, GraphPad Software). A value of $P<0.05$ was considered significant.

Study approval. The study was conducted in an outpatient setting in the Academic Medical Center in Amsterdam, The Netherlands, after approval of the local ethics committee (METC AMC, Amsterdam). All subjects gave informed consent, and our study was in accordance with the Declaration of Helsinki. The trial was conducted in accordance to the original protocol (https://www.trialregister.nl; NTR4785), and the reporting of the trial adheres to the CONSORT guidelines (53). For the skin macrophage analysis, skin biopsies from a similar and previously performed dietary salt intervention study in 12 healthy male subjects were used (https://www.trialregister.nl; NTR4095) (Supplemental Table 3).

\section{Author contributions}

LV and JVDB conceptualized the study. NC, OP, JA, LV, and JVDB designed the methodology. EW, JK, and JVDB contributed to analysis. EW, RW, ES, ER, AN, JB, SV, OP, JK, JVDB, and MPJDW were responsible for investigation. EW, JK, and JVDB wrote the initial draft of the manuscript, and all coauthors participated in review and editing. EW and JVDB were responsible for visualization. LV and JVDB acquired funding. LV and JVDB contributed equally to this work. All authors read and approved the final manuscript. 


\section{Acknowledgments}

JVDB received a VENI grant from ZonMW (91615052), a Netherlands Heart Foundation Junior Postdoctoral grant (2013T003), and Senior Fellowship (2017T048). JK received a VENI grant from ZonMW (91619098). LV was supported by a Dutch Kidney Foundation Junior Kolff grant (KJPB 11.22), Senior Kolff grant (18OKG12), and ZonMW Clinical Fellowship grant (90700310). MPJDW is an Established Investigator of the Netherlands Heart Foundation and is supported by a grant from the Netherlands Heart Foundation and Spark-Holding BV (2015B002), the European Union (ITN-grant EPIMAC and REPROGRAM [EU Horizon 2020]), and the Leducq Transatlantic Network and holds an AMC-fellowship. We acknowledge the support from the Netherlands CardioVascular Research Initiative, Dutch Federation of University Medical Centers, the Netherlands Organization for Health Research and Development, and the Royal Netherlands Academy of Sciences (GENIUS 1 and GENIUS 2; CVON 2011-19 and 2017-20).

Address correspondence to: Liffert Vogt, Department of Internal Medicine, Section of Nephrology, F4-215, Amsterdam UMC, location AMC, Meibergdreef 9 1105AZ Amsterdam, Netherlands. Phone: 31.20.566.9111; Email: 1.vogt@amsterdamumc.nl.

1. Brown IJ, Tzoulaki I, Candeias V, Elliott P. Salt intakes around the world: implications for public health. Int J Epidemiol. 2009;38(3):791-813.

2. He FJ, Li J, Macgregor GA. Effect of longer-term modest salt reduction on blood pressure. Cochrane Database Syst Rev. 2013;(4):CD004937.

3. Lambers Heerspink HJ, Navis G, Ritz E. Salt intake in kidney disease--a missed therapeutic opportunity? Nephrol Dial Transplant. 2012;27(9):3435-3442.

4. Fernández-Ruiz I. Immune system and cardiovascular disease. Nat Rev Cardiol. 2016;13(9):503.

5. Wenzel U, Turner JE, Krebs C, Kurts C, Harrison DG, Ehmke H. Immune Mechanisms in Arterial Hypertension. J Am Soc Nephrol. 2016;27(3):677-686.

6. França CN, et al. Monocyte subtypes and the CCR2 chemokine receptor in cardiovascular disease. Clin Sci. 2017;131(12):1215-1224.

7. Mattson DL. Immune mechanisms of salt-sensitive hypertension and renal end-organ damage. Nat Rev Nephrol. 2019;15(5):290-300.

8. Krane V, Wanner C. Statins, inflammation and kidney disease. Nat Rev Nephrol. 2011;7(7):385-397.

9. Bernelot Moens SJ, et al. Arterial and Cellular Inflammation in Patients with CKD. J Am Soc Nephrol. 2017;28(4):1278-1285.

10. Machnik A, et al. Macrophages regulate salt-dependent volume and blood pressure by a vascular endothelial growth factor-C-dependent buffering mechanism. Nat Med. 2009;15(5):545-552.

11. Chan CT, et al. Reversal of vascular macrophage accumulation and hypertension by a CCR 2 antagonist in deoxycorticosterone/salt-treated mice. Hypertension. 2012;60(5):1207-1212.

12. Zhou X, et al. Variation in dietary salt intake induces coordinated dynamics of monocyte subsets and monocyte-platelet aggregates in humans: implications in end organ inflammation. PLOS ONE. 2013;8(4):e60332.

13. Yi B, et al. Effects of dietary salt levels on monocytic cells and immune responses in healthy human subjects: a longitudinal study. Transl Res. 2015;166(1):103-110.

14. Binger KJ, et al. High salt reduces the activation of IL-4- and IL-13-stimulated macrophages. J Clin Invest. 2015;125(11):4223-4238.

15. Jantsch J, et al. Cutaneous $\mathrm{Na}+$ storage strengthens the antimicrobial barrier function of the skin and boosts macrophage-driven host defense. Cell Metab. 2015;21(3):493-501.

16. Zhang WC, et al. High salt primes a specific activation state of macrophages, M(Na). Cell Res. 2015;25(8):893-910.

17. Titze J, et al. Glycosaminoglycan polymerization may enable osmotically inactive Na+ storage in the skin. Am J Physiol Heart Circ Physiol. 2004;287(1):H203-H208.

18. Selvarajah V, et al. Novel Mechanism for Buffering Dietary Salt in Humans: Effects of Salt Loading on Skin Sodium, Vascular Endothelial Growth Factor C, and Blood Pressure. Hypertension. 2017;70(5):930-937.

19. Tsou CL, et al. Critical roles for CCR2 and MCP-3 in monocyte mobilization from bone marrow and recruitment to inflammatory sites. J Clin Invest. 2007;117(4):902-909.

20. Rorije NMG, et al. Microvascular Permeability after an Acute and Chronic Salt Load in Healthy Subjects: A Randomized Open-label Crossover Intervention Study. Anesthesiology. 2018;128(2):352-360.

21. Fantuzzi L, Borghi P, Ciolli V, Pavlakis G, Belardelli F, Gessani S. Loss of CCR2 expression and functional response to monocyte chemotactic protein (MCP-1) during the differentiation of human monocytes: role of secreted MCP-1 in the regulation of the chemotactic response. Blood. 1999;94(3):875-883.

22. Phillips RJ, Lutz M, Premack B. Differential signaling mechanisms regulate expression of CC chemokine receptor-2 during monocyte maturation. J Inflamm (Lond). 2005;2:14.

23. Larrousse M, Bragulat E, Segarra M, Sierra C, Coca A, de La Sierra A. Increased levels of atherosclerosis markers in salt-sensitive hypertension. Am J Hypertens. 2006;19(1):87-93.

24. Willenborg S, et al. CCR2 recruits an inflammatory macrophage subpopulation critical for angiogenesis in tissue repair. Blood. 2012;120(3):613-625

25. Ishibashi M, et al. Critical role of monocyte chemoattractant protein-1 receptor CCR2 on monocytes in hypertension-induced vascular inflammation and remodeling. Circ Res. 2004;94(9):1203-1210.

26. Boring L, Gosling J, Cleary M, Charo IF. Decreased lesion formation in CCR2-/- mice reveals a role for chemokines in the initiation of atherosclerosis. Nature. 1998;394(6696):894-897. 
27. Maus U, et al. The role of CC chemokine receptor 2 in alveolar monocyte and neutrophil immigration in intact mice. Am $J$ Respir Crit Care Med. 2002;166(3):268-273.

28. Huang DR, Wang J, Kivisakk P, Rollins BJ, Ransohoff RM. Absence of monocyte chemoattractant protein 1 in mice leads to decreased local macrophage recruitment and antigen-specific $\mathrm{T}$ helper cell type 1 immune response in experimental autoimmune encephalomyelitis. J Exp Med. 2001;193(6):713-726.

29. Boring L, et al. Impaired monocyte migration and reduced type 1 (Th1) cytokine responses in C-C chemokine receptor 2 knockout mice. J Clin Invest. 1997;100(10):2552-2561.

30. Lu B, et al. Abnormalities in monocyte recruitment and cytokine expression in monocyte chemoattractant protein 1-deficient mice. J Exp Med. 1998;187(4):601-608.

31. Sakata F, et al. Sodium chloride promotes tissue inflammation via osmotic stimuli in subtotal-nephrectomized mice. Lab Invest. 2017;97(4):432-446

32. Schierke F, et al. Nanomechanics of the endothelial glycocalyx contribute to $\mathrm{Na}^{+}$-induced vascular inflammation. Sci Rep . 2017;7:46476

33. Takahashi $\mathrm{H}$, et al. A high-salt diet enhances leukocyte adhesion in association with kidney injury in young Dahl salt-sensitive rats. Hypertens Res. 2017;40(11):912-920.

34. Selvarajah V, et al. Novel Mechanism for Buffering Dietary Salt in Humans: Effects of Salt Loading on Skin Sodium, Vascular Endothelial Growth Factor C, and Blood Pressure. Hypertension. 2017;70(5):930-937.

35. Müller S, et al. Salt-dependent chemotaxis of macrophages. PLoS ONE. 2013;8(9):e73439.

36. Soehnlein O, Lindbom L, Weber C. Mechanisms underlying neutrophil-mediated monocyte recruitment. Blood. 2009;114(21):4613-4623.

37. Zhou X, Chen K, Lei H, Sun Z. Klotho gene deficiency causes salt-sensitive hypertension via monocyte chemotactic protein-1/ CC chemokine receptor 2-mediated inflammation. J Am Soc Nephrol. 2015;26(1):121-132.

38. Wenzel P, et al. Lysozyme M-positive monocytes mediate angiotensin II-induced arterial hypertension and vascular dysfunction. Circulation. 2011;124(12):1370-1381.

39. Harwani SC. Macrophages under pressure: the role of macrophage polarization in hypertension. Transl Res. 2018;191:45-63.

40. Chung AC, Lan HY. Chemokines in renal injury. J Am Soc Nephrol. 2011;22(5):802-809.

41. Boels MGS, et al. Systemic Monocyte Chemotactic Protein-1 Inhibition Modifies Renal Macrophages and Restores Glomerular Endothelial Glycocalyx and Barrier Function in Diabetic Nephropathy. Am J Pathol. 2017;187(11):2430-2440.

42. Bot I, et al. A novel CCR2 antagonist inhibits atherogenesis in apoE deficient mice by achieving high receptor occupancy. Sci Rep. 2017;7(1):52.

43. Dawson TC, Kuziel WA, Osahar TA, Maeda N. Absence of CC chemokine receptor-2 reduces atherosclerosis in apolipoprotein E-deficient mice. Atherosclerosis. 1999;143(1):205-211.

44. De Ciuceis C, Amiri F, Brassard P, Endemann DH, Touyz RM, Schiffrin EL. Reduced vascular remodeling, endothelial dysfunction, and oxidative stress in resistance arteries of angiotensin II-infused macrophage colony-stimulating factor-deficient mice: evidence for a role in inflammation in angiotensin-induced vascular injury. Arterioscler Thromb Vasc Biol. 2005;25(10):2106-2113.

45. Mallamaci F, Leonardis D, Pizzini P, Cutrupi S, Tripepi G, Zoccali C. Procalcitonin and the inflammatory response to salt in essential hypertension: a randomized cross-over clinical trial. J Hypertens. 2013;31(7):1424-1430.

46. Kurtz TW, DiCarlo SE, Pravenec M, Morris RC. An Appraisal of Methods Recently Recommended for Testing Salt Sensitivity of Blood Pressure. J Am Heart Assoc. 2017;6(4):e005653.

47. Jin X, Kruth HS. Culture of Macrophage Colony-stimulating Factor Differentiated Human Monocyte-derived Macrophages. $J$ Vis Exp. 2016;(112):10.3791/54244.

48. Reiss AB, Siegart NM, De Leon J. Interleukin-6 in atherosclerosis: atherogenic or atheroprotective? Clin Lipidol. 2017;12(1):14-23.

49. Bernelot Moens SJ, et al. PCSK9 monoclonal antibodies reverse the pro-inflammatory profile of monocytes in familial hypercholesterolaemia. Eur Heart J. 2017;38(20):1584-1593.

50. Schneider CA, Rasband WS, Eliceiri KW. NIH Image to ImageJ: 25 years of image analysis. Nat Methods. 2012;9(7):671-675.

51. Barros MH, Hauck F, Dreyer JH, Kempkes B, Niedobitek G. Macrophage polarisation: an immunohistochemical approach for identifying M1 and M2 macrophages. PLoS ONE. 2013;8(11):e80908.

52. Schliefsteiner C, et al. Human Placental Hofbauer Cells Maintain an Anti-inflammatory M2 Phenotype despite the Presence of Gestational Diabetes Mellitus. Front Immunol. 2017;8:888.

53. Moher D, et al. CONSORT 2010 explanation and elaboration: updated guidelines for reporting parallel group randomised trials. BMJ. 2010;340:c869. 